\title{
A geometric solution to the squaring down problem
}

\author{
Lorenzo Ntogramatzidis
}

\begin{abstract}
This paper addresses the problem of the squaring down of LTI systems with the tools of the geometric control theory. More precisely, it is shown how a generic system can be turned into a square and invertible system by means of a state-feedback and an output-injection, and of two static units cascaded at the input and at the output of the given system. In this way, key system properties like phase-minimality, relative degree and infinite zero structure are preserved after the squaring down, and the additional invariant zeros introduced can be arbitrarily assigned in the complex plane.
\end{abstract}

\section{INTRODUCTION}

It is a very well-known fact that several design and synthesis procedures in control theory require that the system or process has an equal number of inputs and outputs and its transfer function matrix is invertible, [7]. Hence, the squaring down problem, consisting of a procedure to turn the system with an unequal number of inputs and outputs into a square and invertible system, has always received a great deal of attention.

The first papers that appeared in the literature on this issue presented a very simple technique for the squaring down, which was based on the introduction of two static units at the input and at the output of the given system, so that the overall system was square and invertible, [8]. It was then noticed, however, that these solutions, led to the inevitable introduction of non assignable additional invariant zeros in the resulting system, [6]. Hence, in the case when any of these new invariant zeros was non minimum-phase, the performance of the closed-loop behaviour was heavily affected.

For this reason, in more recent literature, new solutions based on dynamic compensators cascaded at the input and at the output of the given system have been introduced, with the purpose of ensuring the phase-minimality of the resulting system if the original system was such, [2], [10], [9], [11], [5]. In particular, in [10], [9], the design procedure was carried out for strictly and non strictly proper systems, respectively, by the exploitation of the so-called special coordinate basis for the representation of the plant. In this way, it was proven that these compensators enabled the introduction of additional non minimum phase zeros to be avoided. In the last decade, alternative solutions relying on the use of dynamic pre and post-compensators, have been proposed, all characterized by the property of preserving the

L. Ntogramatzidis is with the Dipartimento di Elettronica, Informatica e Sistemistica, Università di Bologna, viale Risorgimento, 2 - 40136 Bologna, Italy Intogramatzidis@deis.unibo.it

D. Prattichizzo is with the Dipartimento di Ingegneria dell' Informazione, Università di Siena, via Roma, 77 - 53100 Siena, Italy prattichizzo@dii.unisi.it

\author{
Domenico Prattichizzo
}

phase-minimality of the plant, but presenting different kinds of advantages, see e.g., [7] [11], [5], and references therein. The procedure proposed in this paper provides a geometric solution to this problem, which is based on the introduction of static compensators at the input and at the output of the given system, working jointly with an algebraic statefeedback and output-injection units, whose purpose is that of arbitrarily assigning all the additional invariant zeros introduced in the squared-down system. As a result, the compensation scheme herein proposed preserves the simplicity of the static compensators, but avoids the introduction of possibly non minimum-phase zeros. First, the design of a left-inverter will be presented, based on a static pre-compensator and an algebraic state-feedback term such that the system thus obtained is left-invertible and the additional invariant zeros are all assignable in the complex plane; moreover, if the given system is right-invertible, so is the resulting system, and the relative degree does not change after the squaring down: hence, the functional controllability properties of the original system are preserved. Furthermore, the infinite zero structure is also preserved. Clearly, a straightforward dualization of the aforementioned procedure yields a rightinverter which is based on a static post-compensator and an algebraic output-injection, thus preserving the functional observability of the plant and the additional invariant zeros of the resulting system can be arbitrarily assigned in the complex plane.

The advantages of the proposed approach are manifold. On the one hand, the geometric setting herein employed is coordinate-free in nature, and leads to intuitive results without the need of resorting to changes of basis which, as shown in [5], are not computationally robust. On the other side, this method yields a new insight into the geometric structure of LTI systems, generalizing the properties presented in [3] for non strictly proper systems. As a consequence, several ancillary results of independent interest on the geometric structure of LTI systems will be derived. Furthermore, the possibility of deriving separately a left and a right-inverter furnishes useful tools in many optimization problems. For instance, the infinite-horizon LQ problem will be investigated in the discrete-time case: when the underlying system is non left-invertible, the discrete algebraic Riccati equation does not admit a stabilizing solution. On the other hand, by performing the left-inversion of the system by means of the procedure herein described, a new system is obtained such that the discrete ARE has a stabilizing solution, which yields the set of all optimal feedback gains parametrized in terms of the eigenvalues that can be assigned in the outputnulling reachability subspace. Furthermore, this method also 
provides a non-iterative algorithm for the solution of the generalized discrete ARE.

Notation. Throughout this paper, we denote by $\mathbb{N}$ the positive integers including zero, and by $\mathbb{N}^{*}$ the strictly positive integers. The symbol $0_{n}$ denotes the origin of the vector space $\mathbb{R}^{n}$. The image and the kernel of matrix $A \in \mathbb{R}^{n \times m}$ are denoted by $\operatorname{im} A$ and $\operatorname{ker} A$. Given a subspace $\mathscr{Y}$ of $\mathbb{R}^{n}$, the symbol $A^{-1} \mathscr{Y}$ stands for the inverse image of $\mathscr{Y}$ with respect to the linear map $A$, while $\mathscr{Y}^{\perp}$ represents the orthogonal complement of $\mathscr{Y}$. Denote by $A^{\top}$ and by $A^{\ddagger}$ the transpose and the Moore-Penrose pseudo-inverse of $A$, respectively. The symbol $\sigma(A)$ denotes the spectrum of $A$. The restriction of a linear map $A$ to the $A$-invariant subspace $\mathscr{J}$ is written $\left.A\right|_{\mathscr{J}}$; the eigenvalues of $A$ restricted to $\mathscr{J}$ are denoted by $\left.\sigma(A)\right|_{\mathscr{J}}$. If $\mathscr{J}_{1}$ and $\mathscr{J}_{2}$ are $A$-invariant subspaces and $\mathscr{J}_{1} \subseteq \mathscr{J}_{2}$, the mapping induced by $A$ on the quotient space $\mathscr{J}_{2} / \mathscr{J}_{1}$ is denoted by $\left.A\right|_{\mathscr{J}_{2} / \mathscr{J}_{1}}$.

\section{Problem Statement}

Consider a LTI continuous-time system $\Sigma$ described by

$$
\begin{aligned}
& \dot{x}(t)=A x(t)+B u(t), \\
& y(t)=C x(t)+D u(t),
\end{aligned}
$$

where, for all $t \geq 0, x(t) \in \mathbb{R}^{n}$ is the state, $u(t) \in \mathbb{R}^{m}$ is the control input, $y(t) \in \mathbb{R}^{p}$ is the output, $A, B, C$ and $D$ are real constant matrices of suitable sizes. With no loss of generality it is assumed that $\left[\begin{array}{cc}B^{\top} & D^{\top}\end{array}\right]^{\top}$ has linearly independent columns and $\left[\begin{array}{cc}C & D\end{array}\right]$ has linearly independent rows. We concisely identify $\Sigma$ with the quadruple $(A, B, C, D)$.

The aim of this paper is that of presenting a design procedure based on geometric techniques for the squaring down of the given system $\Sigma$, i.e., a transformation involving $\Sigma$, so as to obtain a new system $\Sigma_{\mathrm{sq}}$ having the same number of inputs and outputs and such that its transfer function matrix is invertible over the set of rational matrices.

\section{GEOMETRIC PRELIMINARIES}

For the readers' convenience, in this section some fundamental definitions and results of the geometric approach which will be used in the sequel are recalled (for a detailed discussion on the topics herein introduced we refer to [4], [12], [13]). First, we define an output-nulling subspace $\mathscr{V}_{\Sigma}$ of $\Sigma$ as a subspace of $\mathbb{R}^{n}$ satisfying

$$
\left[\begin{array}{l}
A \\
C
\end{array}\right] \mathscr{V}_{\Sigma} \subseteq\left(\mathscr{V}_{\Sigma} \times 0_{p}\right)+i m\left[\begin{array}{c}
B \\
D
\end{array}\right]
$$

The set of output-nulling subspaces of $\Sigma$ is closed with respect to subspace addition. Thus, the sum of all the outputnulling subspaces of $\Sigma$ is the largest output-nulling subspace of $\Sigma$, and will be herein denoted by $\mathscr{V}_{\Sigma}^{*}$. Clearly, in the case when $D$ is zero, $\mathscr{V}_{\Sigma}^{*}$ coincides with the maximal $(A, B)$ controlled invariant subspace contained in the null-space of matrix $C$, [4]. In the following lemmas, the most important properties of the output-nulling subspaces are presented.
Lemma 1: The subspace $\mathscr{V}_{\Sigma}$ is output-nulling for $\Sigma$ iff a matrix $F \in \mathbb{R}^{m \times n}$ exists such that

$$
\begin{aligned}
& (A+B F) \mathscr{V}_{\Sigma} \subseteq \mathscr{V}_{\Sigma}, \\
& \mathscr{V}_{\Sigma} \subseteq \operatorname{ker}(C+D F) .
\end{aligned}
$$

Moreover, the subspace $\mathscr{V}_{\Sigma}^{*}$ is the largest subspace for which a matrix $F \in \mathbb{R}^{m \times n}$ exists such that (3) hold.

Lemma 2: The sequence of subspaces $\left(\mathscr{V}_{\Sigma}^{i}\right)_{i \in \mathbb{N}}$ described by the recurrence

$$
\begin{aligned}
& \mathscr{V}_{\Sigma}^{0}=\mathbb{R}^{n}, \\
& \mathscr{V}_{\Sigma}^{i}=\left[\begin{array}{l}
A \\
C
\end{array}\right]^{-1}\left(\left(\mathscr{V}_{\Sigma}^{i-1} \times 0_{p}\right)+i m\left[\begin{array}{l}
B \\
D
\end{array}\right]\right), \quad i \in \mathbb{N}^{*}
\end{aligned}
$$

is monotonically non-increasing. Moreover, there exists $k \leq n-1$ such that $\mathscr{V}_{\Sigma}^{k+1}=\mathscr{V}_{\Sigma}^{k}$. For this $k$ there holds $\mathscr{V}_{\Sigma}^{*}=\mathscr{V}_{\Sigma}^{k}$

A matrix $F$ satisfying (3) is generally referred to as a friend of $\mathscr{V}_{\Sigma}$. The dual concept is the input-containing subspace: a subspace $\mathscr{S}_{\Sigma}$ is said to be input-containing if it satisfies

$$
\left[\begin{array}{ll}
A & B
\end{array}\right]\left(\left(\mathscr{S}_{\Sigma} \times \mathbb{R}^{m}\right) \cap \operatorname{ker}\left[\begin{array}{ll}
C & D
\end{array}\right]\right) \subseteq \mathscr{S}_{\Sigma} .
$$

The set of all input-containing subspaces of $\Sigma$ is a closed with respect to the subspace intersection. Thus, the intersection of all input-containing subspaces of $\Sigma$ is the smallest inputcontaining subspace of $\Sigma$, and will be denoted by $\mathscr{S}_{\Sigma}^{*}$.

Lemma 3: The subspace $\mathscr{S}_{\Sigma}$ is input-containing for $\Sigma$ iff a matrix $G \in \mathbb{R}^{n \times p}$ exists such that

$$
\begin{aligned}
& (A+G C) \mathscr{S}_{\Sigma} \subseteq \mathscr{S}_{\Sigma}, \\
& \mathscr{S}_{\Sigma} \supseteq \operatorname{im}(B+G D) .
\end{aligned}
$$

Moreover, the subspace $\mathscr{S}_{\Sigma}^{*}$ is the smallest subspace for which a matrix $G \in \mathbb{R}^{n \times p}$ exists such that (6) hold.

Lemma 4: The sequence of subspaces $\left(\mathscr{S}_{\Sigma}^{i}\right)_{i \in \mathbb{N}}$ described by the recurrence

$\mathscr{S}_{\Sigma}^{0}=0_{n}$
$\mathscr{S}_{\Sigma}^{i}=\left[\begin{array}{ll}A & B\end{array}\right]\left(\left(\mathscr{S}_{\Sigma}^{i-1} \times \mathbb{R}^{m}\right) \cap k e r\left[\begin{array}{ll}C & D\end{array}\right]\right), \quad i \in \mathbb{N}^{*}$

is monotonically non-decreasing. Moreover, there exists $k \leq n-1$ such that $\mathscr{S}_{\Sigma}^{k+1}=\mathscr{S}_{\Sigma}^{k}$. For this $k$ there holds $\mathscr{S}_{\Sigma}^{*}=\mathscr{S}_{\Sigma}^{k}$.

Let $F \in \mathbb{R}^{m \times n}$ be a friend of the output-nulling subspace $\mathscr{V}_{\Sigma}$. The output-nulling reachability subspace $\mathscr{R}_{\Sigma}$ on $\mathscr{V}_{\Sigma}$ is the smallest $(A+B F)$-invariant subspace of $\mathbb{R}^{n}$ containing $\mathscr{V}_{\Sigma} \cap B \operatorname{ker} D$. We denote by $\mathscr{R}_{\Sigma}^{*}$ the output-nulling reachability subspace on $\mathscr{V}_{\Sigma}^{*}$. The following relation holds:

$$
\mathscr{R}_{\Sigma}^{*}=\mathscr{V}_{\Sigma}^{*} \cap \mathscr{S}_{\Sigma}^{*}
$$

A proof of this fact for systems with direct feedthrough can be found in [12], Theorem 8.22. The subspace $\mathscr{R}_{\Sigma}^{*}$ can be thought as the locus of all the initial states that are reachable in finite time from the origin while mantaining the corresponding output equal to zero.

It is a well-known and easily established fact that, for any friend $F$ of $\mathscr{V}_{\Sigma}^{*}, F$ is also a friend of $\mathscr{R}_{\Sigma}^{*}$. Given a friend $F$ of $\mathscr{V}_{\Sigma}^{*}$, the eigenvalues of $(A+B F)$ restricted to $\mathscr{V}_{\Sigma}^{*}$, i.e. $\left.\sigma(A+B F)\right|_{\mathscr{V}_{\Sigma}^{*}}$, are split into two sets: the eigenvalues of 
$\left.(A+B F)\right|_{\mathscr{R}_{\Sigma}^{*}}$ are all freely assignable by a suitable choice of the friend $F$ of $\mathscr{V}_{\Sigma}^{*}$. The eigenvalues of $\left.(A+B F)\right|_{\mathscr{V}_{\Sigma}^{*} / \mathscr{R}_{\Sigma}^{*}}$ do not depend on the choice of the friend $F$ of $\mathscr{V}_{\Sigma}^{*}$. Its elements are the invariant zeros of $\Sigma$, [1]. In symbols

$$
\mathscr{Z}(\Sigma)=\left.\sigma(A+B F)\right|_{\mathscr{V}_{\Sigma}^{*}} \mid \mathscr{R}_{\Sigma}^{*} \cdot
$$

Given a friend $G$ of $\mathscr{S}_{\Sigma}^{*}$, the eigenvalues of $(A+$ $G C)\left.\right|_{\mathbb{R}^{n} / \mathscr{V}_{\Sigma}^{*}+\mathscr{S}_{\Sigma}^{*}}$ are all freely assignable by a suitable choice of $G$, while the eigenvalues of $\left.(A+G C)\right|_{\mathscr{V}_{\Sigma}^{*}+\mathscr{S}_{\Sigma}^{*} / \mathscr{S}_{\Sigma}^{*}}$ are fixed, and are the invariant zeros of $\Sigma$.

Definition 1: The system $\Sigma$ is said to be left-invertible if the matrix $\left[\begin{array}{ll}B^{\top} & D^{\top}\end{array}\right]^{\top}$ is monic and $\mathscr{V}_{\Sigma}^{*} \cap \mathscr{S}_{\Sigma}^{*}=0_{n}$.

Stated differently, we may say that $\Sigma$ is left-invertible if no pair of distinct input functions give rise to the same output function with zero initial condition. The following theorem provides necessary and sufficient conditions for leftinvertibility.

Lemma 5: System $\Sigma$ is left-invertible iff any of the following statements hold true:

1) the map $\left[B^{\top} D^{\top}\right]^{\top}$ is monic and $\mathscr{V}_{\Sigma}^{*} \cap B \operatorname{ker} D=0_{n}$;

2) the map $\left[B^{\top} D^{\top}\right]^{\top}$ is monic and $B^{-1} \mathscr{V}_{\Sigma}^{*} \cap \operatorname{ker} D=0_{m}$.

Definition 2: The system $\Sigma$ is said to be right-invertible if the matrix $\left[\begin{array}{ll}C & D\end{array}\right]$ is epic and $\mathscr{V}_{\Sigma}^{*}+\mathscr{S}_{\Sigma}^{*}=\mathbb{R}^{n}$.

Stated differently, we may say that $\Sigma$ is right-invertible if for any arbitrarily assigned impulsive-smooth output, an impulsive-smooth input exists which yields that output with zero initial condition.

Lemma 6: System $\Sigma$ is right-invertible iff any of the following statements hold true:

1) the map $\left[\begin{array}{ll}C & D\end{array}\right]$ is epic and $\mathscr{S}_{\Sigma}^{*}+C^{-1} \operatorname{im} D=\mathbb{R}^{n}$;

2) the map $\left[\begin{array}{ll}C & D\end{array}\right]$ is epic and $C \mathscr{S}_{\Sigma}^{*}+i m D=\mathbb{R}^{p}$.

Consider the sequence $\left(\mathscr{S}_{\Sigma}^{i}\right)_{i \in \mathbb{N}}$ described by (7). We define the relative degree of the right-invertible system $\Sigma$ as the least integer $k \in \mathbb{N}$ such that $\mathscr{V}_{\Sigma}^{*}+\mathscr{S}^{k+1}=\mathbb{R}^{n}$. Finally, let $v_{i}:=\operatorname{dim}\left(\mathscr{V}_{\Sigma}^{*}+\mathscr{S}^{i}\right)-\operatorname{dim}\left(\mathscr{V}_{\Sigma}^{*}+\mathscr{S}^{i-1}\right)$, for $i \in \mathbb{N}$. Let $\rho_{i}:=$ $\operatorname{card}\left\{j \in \mathbb{N} \mid v_{j} \geq i\right\}$. The $\rho_{i}$ are the orders of the zeros at infinity of $\Sigma$, see [1, Theorem 4].

\section{DESIGN OF A LEFT-INVERTER}

Consider a non left-invertible system $\Sigma$. The first step in the construction of a squaring down procedure consists of a transformation on $\Sigma$, involving an algebraic state-feedback and an input static unit, so that the system thus obtained, say $\widehat{\Sigma}$, is left-invertible, see Fig. 1. By performing a

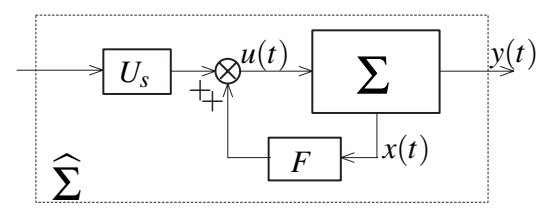

Fig. 1. Block diagram of a left-inverter

state-feedback with a friend $F$ of $\mathscr{V}_{\Sigma}^{*}$ assigning arbitrarily all the eigenvalues of $\left.(A+B F)\right|_{\mathscr{R}_{\Sigma}^{*}}$, and by eliminating all the input functions yielding motions on $\mathscr{R}_{\Sigma}^{*}$, we obtain a new system $\widehat{\Sigma}$ whose output-nulling reachable subspace $\mathscr{R}_{\widehat{\Sigma}}^{*}$ is the origin, and its invariant zeros are those of the original system, plus those assigned through $F$. It follows in particular that if $\Sigma$ is minimum-phase, the modified system $\widehat{\Sigma}$ remains such with a suitable choice of $F$.

These results are presented and proved in the following theorem.

Theorem 1: Let $F$ be a friend of $\mathscr{V}_{\Sigma}^{*}$. Let $U_{s}$ be a basis matrix of the subspace $\mathscr{U}_{s}:=\left(B^{-1} \mathscr{V}_{\Sigma}^{*} \cap k e r D\right)^{\perp}$, whose dimension is denoted by $s$. Consider the quadruple $\widehat{\Sigma}:=(A+$ $\left.B F, B U_{s}, C+D F, D U_{s}\right)$. The following results hold:

1) $\mathscr{V}_{\Sigma}^{*}=\mathscr{V}_{\widehat{\Sigma}}^{*}$

2) $\widehat{\Sigma}$ is left-invertible;

3) $\mathscr{Z}(\widehat{\Sigma})=\left.\mathscr{Z}(\Sigma) \uplus \sigma(A+B F)\right|_{\mathscr{R}_{\Sigma}^{*}}$.

Proof: First, we denote by $\Sigma_{F}$ the quadruple $(A+B F, B, C+D F, D)$. A straightforward computation show that an output-nulling subspace of $\Sigma$ is also outputnulling for $\Sigma_{F}$ (and viceversa), i.e., $\mathscr{V}_{\Sigma}^{*}=\mathscr{V}_{\Sigma_{F}}^{*}$, [12, p.169]. Now, we prove that any output-nulling subspace $\mathscr{V}_{\Sigma_{F}}$ of $\Sigma_{F}$ is output-nulling for $\widehat{\Sigma}$ and viceversa. We start by proving the following two identities:

$$
\begin{aligned}
& \mathscr{V}_{\Sigma_{F}^{*}}^{*}+i m B=\mathscr{V}_{\Sigma_{F}}^{*}+i m B U_{s}, \\
& i m D=i m D U_{s} .
\end{aligned}
$$

Apply the linear map $B$ to both sides of the trivial identity $\mathbb{R}^{m}=\left(B^{-1} \mathscr{V}_{\Sigma}^{*} \cap \operatorname{ker} D\right) \oplus \mathscr{U}_{s}$ to obtain

$$
\begin{aligned}
\operatorname{im} B & =\left(\mathscr{V}_{\Sigma}^{*} \cap B \operatorname{ker} D\right)+\operatorname{im} B U_{s} \\
& =\left(\mathscr{V}_{\Sigma_{F}}^{*} \cap B \operatorname{ker} D\right)+\operatorname{im} B U_{s}
\end{aligned}
$$

By adding $\mathscr{V}_{\Sigma_{F}}$ to both sides of the former, we get (9), since $\mathscr{V}_{\Sigma_{F}} \supseteq \mathscr{R}_{\Sigma_{F}}^{*} \supseteq \mathscr{V}_{\Sigma_{F}}^{*} \cap B$ kerD. In order to prove (10), note that

$$
\begin{aligned}
i m D U_{s} & =i m D\left(\left(B^{-1} \mathscr{V}_{\Sigma}^{*}\right)^{\perp}+(k e r D)^{\perp}\right) \\
& =i m D\left(B^{-1} \mathscr{V}_{\Sigma}^{*}\right)^{\perp}+i m D D^{\top}=i m D .
\end{aligned}
$$

Equations (9) and (10) yield the identity

$$
\left(\mathscr{V}_{\Sigma_{F}} \times 0_{p}\right)+i m\left[\begin{array}{c}
B \\
D
\end{array}\right]=\left(\mathscr{V}_{\Sigma_{F}} \times 0_{p}\right)+i m\left(\left[\begin{array}{l}
B \\
D
\end{array}\right] U_{s}\right),
$$

which leads to the following inclusion:

$$
\left[\begin{array}{l}
A+B F \\
C+D F
\end{array}\right] \mathscr{N}_{\Sigma_{F}} \subseteq\left(\mathscr{V}_{\Sigma_{F}} \times 0_{p}\right)+i m\left(\left[\begin{array}{c}
B \\
D
\end{array}\right] U_{S}\right) .
$$

Hence, any output-nulling subspace of $\Sigma_{F}$ is output-nulling for $\widehat{\Sigma}$, as well. Conversely, if $\mathscr{V}_{\Sigma}$ is output-nulling for $\widehat{\Sigma}$, it follows that it is output-nulling for $\Sigma_{F}$. In fact, in general

$$
\operatorname{im}\left(\left[\begin{array}{c}
B \\
D
\end{array}\right] U_{s}\right) \subseteq i m\left[\begin{array}{l}
B \\
D
\end{array}\right] .
$$

We may therefore conclude that any output-nulling subspace of $\Sigma$ is also output-nulling of $\widehat{\Sigma}$. As a result, $\mathscr{V}_{\Sigma}^{*}=\mathscr{V}_{\widehat{\Sigma}}^{*}$.

Now, by taking Lemma 5 into account, we show that $\widehat{\Sigma}$ is left-invertible by proving that $\left(B U_{s}\right)^{-1} \mathscr{V}_{\widehat{\Sigma}}^{*} \cap \operatorname{ker}\left(D U_{s}\right)=0_{s}$. Let $\quad \omega \in\left(B U_{s}\right)^{-1} \mathscr{V}_{\widehat{\Sigma}}^{*} \cap \operatorname{ker}\left(D U_{s}\right)$. Thus, $\quad B U_{s} \omega \in \mathscr{V}_{\widehat{\Sigma}}^{*}$ and $D U_{s} \omega=0_{p}$. Define $\tilde{\omega}:=U_{s} \omega$. The former yields 
$\tilde{\omega} \in B^{-1} \mathscr{V}_{\widehat{\Sigma}}^{*} \cap \operatorname{ker} D=\mathscr{U}_{s}^{\perp}$, since $\mathscr{V}_{\Sigma}^{*}=\mathscr{V}_{\widehat{\Sigma}}^{*}$. However, by definition $\tilde{\omega}$ lies in the range of $U_{s}$, hence it belongs to $\left(B^{-1} \mathscr{V}_{\Sigma}^{*} \cap k e r D\right)^{\perp}$. It follows that $\tilde{\omega}=0_{m}$. From the injectivity of $U_{s}$, we may conclude that $\omega=0_{s}$. As a result, $\widehat{\Sigma}$ is left-invertible.

Finally, by definition of invariant zeros there holds $\mathscr{Z}(\widehat{\Sigma})=\left.\sigma(A+B F)\right|_{\mathscr{V}_{\widehat{\Sigma}}^{*} / \mathscr{R}_{\widehat{\Sigma}}^{*}}$, which equals $\left.\sigma(A+B F)\right|_{\mathscr{V}_{\widehat{\Sigma}}^{*}}$ since $\widehat{\Sigma}$ is left-invertible. However, since $\mathscr{V}_{\Sigma}^{*}=\mathscr{V}_{\widehat{\Sigma}}^{*}$, we get

$$
\begin{aligned}
\mathscr{Z}(\widehat{\Sigma}) & =\left.\left.\sigma(A+B F)\right|_{\mathscr{V}_{\Sigma}^{*} / \mathscr{R}_{\Sigma}^{*}} \uplus \sigma(A+B F)\right|_{\mathscr{R}_{\Sigma}^{*}} \\
& =\left.\mathscr{Z}(\Sigma) \uplus \sigma(A+B F)\right|_{\mathscr{R}_{\Sigma}^{*}} .
\end{aligned}
$$

The transformation described in Theorem 1 enjoys another important property: it does not reduce the functional controllability properties of the original system, i.e., if $\Sigma$ is right-invertible, so is the resulting system $\widehat{\Sigma}$ (and viceversa). Even more is true: the subspace $\mathscr{V}_{\Sigma}^{*}+\mathscr{S}_{\Sigma}^{*}$ is invariant under the transformation presented in Theorem 1. As already noted, this subspace is dual to $\mathscr{R}_{\Sigma}^{*}=\mathscr{V}_{\Sigma}^{*} \cap \mathscr{S}_{\Sigma}^{*}$. This result is presented and proved in the following proposition.

Proposition 1: Let $F$ be a friend of $\mathscr{V}_{\Sigma}^{*}$. Let $U_{s}$ be a basis matrix of the subspace $\mathscr{U}_{s}:=\left(B^{-1} \mathscr{V}_{\Sigma}^{*} \cap \operatorname{ker} D\right)^{\perp}$, whose dimension is denoted by $s$. Consider the sequence of subspaces $\left(\mathscr{S}_{\Sigma}^{i}\right)_{i \in \mathbb{N}}$. The following equality holds

$$
\mathscr{V}_{\Sigma}^{*}+\mathscr{S}_{\Sigma}^{i}=\mathscr{V}_{\widehat{\Sigma}}^{*}+\mathscr{S}_{\widehat{\Sigma}}^{i}, \quad i \in \mathbb{N} .
$$

Hence, in particular, $\Sigma$ is right-invertible iff $\widehat{\Sigma}$ is such. If this is the case, the relative degree of $\widehat{\Sigma}$ and that of $\Sigma$ are equal. Moreover, $\widehat{\Sigma}$ and $\Sigma$ have the same zero structure at infinity. Proof: Denote by $\bar{\Sigma}$ the quadruple $\left(A, B U_{s}, C, D U_{s}\right)$. We prove that $\mathscr{V}_{\Sigma}^{*}+\mathscr{S}_{\Sigma}^{i}=\mathscr{V}_{\bar{\Sigma}}^{*}+\mathscr{S}_{\bar{\Sigma}}^{i}$. To this aim, consider the two sequences of subspaces $\left(\mathscr{V}_{\Sigma}^{i}\right)_{i \in \mathbb{N}}$ and $\left(\mathscr{V}_{\bar{\Sigma}}^{i}\right)_{i \in \mathbb{N}}$ described in (4), which respectively converge to $\mathscr{V}_{\Sigma}^{*}$ and $\mathscr{V}_{\bar{\Sigma}}^{*}$ in at most $n-1$ steps. We first verify by induction that for any $i \in \mathbb{N}$ we have $\mathscr{V}_{\Sigma}^{i}=\mathscr{V}_{\bar{\Sigma}}^{i}$. This fact is clearly true when $i=0$. Let us now suppose that it holds for a given $i-1$, i.e., $\mathscr{V}_{\Sigma}^{i-1}=\mathscr{V}_{\bar{\Sigma}}^{i-1}$, and let us prove the same fact for $i$, i.e., $\mathscr{V}_{\Sigma}^{i}=\mathscr{V}_{\bar{\Sigma}}^{i}$. The following inclusion, that will be useful in the sequel, holds:

$$
\left[\begin{array}{c}
B \\
D
\end{array}\right] \mathscr{U}_{s}^{\perp} \subseteq\left(\mathscr{V}_{\Sigma}^{*} \cap B \operatorname{ker} D\right) \times 0_{p} \subseteq \mathscr{V}_{\Sigma}^{i-1} \times 0_{p} .
$$

In fact, since sequence (4) is monotonically non-increasing, $\mathscr{V}_{\Sigma}^{i-1} \supseteq \mathscr{V}_{\Sigma}^{*} \supseteq \mathscr{V}_{\Sigma}^{*} \cap B$ kerD. Now, from (4) we find

$$
\begin{aligned}
\mathscr{V}_{\Sigma}^{i} & =\left[\begin{array}{l}
A \\
C
\end{array}\right]^{-1}\left(\left(\mathscr{V}_{\Sigma}^{i-1} \times 0_{p}\right)+i m\left[\begin{array}{l}
B \\
D
\end{array}\right]\left(\mathscr{U}_{s}^{\perp} \oplus \mathscr{U}_{s}\right)\right) \\
& =\left[\begin{array}{l}
A \\
C
\end{array}\right]^{-1}\left(\left(\mathscr{V}_{\bar{\Sigma}}^{i-1} \times 0_{p}\right)+\left[\begin{array}{c}
B \\
D
\end{array}\right] \mathscr{U}_{s}\right)
\end{aligned}
$$

where the last equality is a consequence of (11). Hence, indeed $\mathscr{V}_{\Sigma}^{i}=\mathscr{V}_{\bar{\Sigma}}^{i}$. Now, we prove by induction that for any $i \in \mathbb{N}, \mathscr{S}_{\Sigma}^{i} \supseteq \mathscr{S}_{\bar{\Sigma}}^{i}$. Clearly, $\mathscr{S}_{\Sigma}^{0}=\mathscr{S}_{\bar{\Sigma}}^{0}$. Let us suppose that $\mathscr{S}_{\Sigma}^{i-1} \supseteq \mathscr{S}_{\bar{\Sigma}}^{i-1}$ for a given $i$, and let us prove that $\mathscr{S}_{\Sigma}^{i} \supseteq \mathscr{S}_{\bar{\Sigma}}^{i}$. Let $\xi \in \mathscr{S}_{\bar{\Sigma}}^{i}$. By (5), it follows that $\xi_{1} \in \mathscr{S}_{\bar{\Sigma}}^{i-1} \subseteq \mathscr{S}_{\Sigma}^{i-1}$ and $\xi_{2} \in \mathbb{R}^{s}$ exist such that $C \xi_{1}+D U_{s} \xi_{2}=0_{p}$ and $A \xi_{1}+B U_{s} \xi_{2}=\xi$. Define $\xi_{2}^{\prime}:=U_{s} \xi_{2}$.
It follows that $\xi_{1} \in \mathscr{S}_{\Sigma}^{i-1}, C \xi_{1}+D \xi_{2}^{\prime}=0$, and $A \xi_{1}+B \xi_{2}^{\prime}=\xi$. The latter three relations imply that $\xi \in \mathscr{S}_{\Sigma}^{i}$. Hence, we have shown that for any $i \in \mathbb{N}$ we have $\mathscr{S}_{\Sigma}^{i} \supseteq \mathscr{S}_{\bar{\Sigma}}^{i}$ and $\mathscr{V}_{\Sigma}^{i}=\mathscr{V}_{\bar{\Sigma}}^{i}$. A direct consequence of this result is that for any $i \in \mathbb{N}$, $\mathscr{V}_{\Sigma}^{i}+\mathscr{S}_{\Sigma}^{i} \supseteq \mathscr{V}_{\bar{\Sigma}}^{i}+\mathscr{S}_{\bar{\Sigma}}^{i}$. In order to show that the converse inclusion is true, i.e., that $\mathscr{V}_{\Sigma}^{i}+\mathscr{S}_{\Sigma}^{i} \subseteq \mathscr{V}_{\bar{\Sigma}}^{i}+\mathscr{S}_{\bar{\Sigma}}^{i}$, we proceed by induction again. The inclusion holds for $i=0$. Let us suppose that $\mathscr{V}_{\Sigma}^{i-1}+\mathscr{S}_{\Sigma}^{i-1} \subseteq \mathscr{V}_{\bar{\Sigma}}^{i-1}+\mathscr{S}_{\bar{\Sigma}}^{i-1}$ for some defined $i$, and let us prove that $\mathscr{V}_{\Sigma}^{i}+\mathscr{S}_{\Sigma}^{i} \subseteq \mathscr{V}_{\bar{\Sigma}}^{i}+\mathscr{S}_{\bar{\Sigma}}^{i}$. Consider an arbitrary $\xi \in \mathscr{V}_{\Sigma}^{i}+\mathscr{S}_{\Sigma}^{i}$. It follows that $\xi_{1} \in \mathscr{S}_{\Sigma}^{i-1}, \xi_{2} \in \mathbb{R}^{m}$ and $\xi_{3} \in \mathscr{V}_{\Sigma}^{i}$ exist such that $C \xi_{1}+D \xi_{2}=0_{p}$ and $A \xi_{1}+B \xi_{2}+\xi_{3}=\xi$. Two vectors $\xi_{2}^{\prime} \in \mathscr{U}_{s}$ and $\xi_{2}^{\prime \prime} \in \mathscr{U}_{s}^{\perp}$ exist such that $\xi_{2}=\xi_{2}^{\prime}+\xi_{2}^{\prime \prime}$. Hence, $B \xi_{2}^{\prime \prime} \in \mathscr{V}_{\Sigma}^{*}$ and $D \xi_{2}^{\prime \prime}=0_{p}$. By linearity there holds

$$
\xi=\left[\begin{array}{ll}
A & B
\end{array}\right]\left[\begin{array}{c}
\xi_{1} \\
\xi_{2}^{\prime}+\xi_{2}^{\prime \prime}
\end{array}\right]+\xi_{3}=\left[\begin{array}{ll}
A & B
\end{array}\right]\left[\begin{array}{l}
\xi_{1} \\
\xi_{2}^{\prime}
\end{array}\right]+\tilde{\xi}+\xi_{3},
$$

where $\tilde{\xi}:=\left[\begin{array}{ll}A & B\end{array}\right]\left[\begin{array}{c}0_{n} \\ \xi_{2}^{\prime \prime}\end{array}\right]$. On the other hand, as already observed, $D \xi_{2}^{\prime \prime}=0_{p}$. It follows that $C \xi_{1}+D \xi_{2}=C \xi_{1}+D \xi_{2}^{\prime}=0_{p}$. Moreover, since $\xi_{2}^{\prime} \in \mathscr{U}_{s}$, a vector $\bar{\xi}_{2} \in \mathbb{R}^{s}$ exists such that $\xi_{2}^{\prime}=U_{s} \bar{\xi}_{2}$. Hence $C \xi_{1}+D U_{s} \bar{\xi}_{2}=0_{p}$ and, since $\mathscr{S}_{\Sigma}^{i-1}=\mathscr{S}_{\bar{\Sigma}}^{i-1}$, we find

$$
\left[\begin{array}{l}
\xi_{1} \\
\bar{\xi}_{2}
\end{array}\right] \in\left(\mathscr{S}_{\bar{\Sigma}}^{i-1} \times \mathbb{R}^{s}\right) \cap \operatorname{ker}\left[\begin{array}{ll}
C & D U_{s}
\end{array}\right] .
$$

Finally, $\quad \xi^{*}:=\tilde{\xi}+\xi_{3}=B \xi_{2}^{\prime \prime}+\xi_{3} \in \mathscr{V}_{\Sigma}^{*}+\mathscr{V}_{\Sigma}^{i}=\mathscr{V}_{\Sigma}^{i} . \quad$ We have shown that $\xi_{1} \in \mathscr{S}_{\bar{\Sigma}}^{i-1}, \bar{\xi}_{2} \in \mathbb{R}^{s}$ and $\xi_{3} \in \mathscr{V}_{\bar{\Sigma}}^{i}$ exist such that $A \xi_{1}+B U_{s} \bar{\xi}_{2}+\xi_{3}=\xi$ and $C \xi_{1}+D U_{s} \bar{\xi}_{2}=0_{p}$. This implies that $\xi \in \mathscr{V}_{\bar{\Sigma}}^{i}+\mathscr{S}_{\bar{\Sigma}}^{i}$. Hence, we have indeed $\mathscr{V}_{\Sigma}^{*}+\mathscr{S}_{\Sigma}^{*}=\mathscr{V}_{\bar{\Sigma}}^{*}+\mathscr{S}_{\bar{\Sigma}}^{*}$. Since $\mathscr{V}_{\Sigma}^{*}$ and $\mathscr{S}_{\Sigma}^{*}$ are invariant under state feedback, [12, p.169, p.183], it follows that $\mathscr{V}_{\Sigma}^{*}+\mathscr{S}_{\Sigma}^{*}=\mathscr{V}_{\Sigma_{F}}^{*}+\mathscr{S}_{\Sigma_{F}}^{*}$, where $\Sigma_{F}$ is defined as in the proof of Theorem 1. Hence, the procedure outlined above can be applied to $\Sigma_{F}$, yielding $\mathscr{V}_{\Sigma}^{*}+\mathscr{S}_{\Sigma}^{*}=\mathscr{V}_{\Sigma_{F}^{*}}^{*}+\mathscr{S}_{\Sigma_{F}^{*}}^{*}=\mathscr{V}_{\widehat{\Sigma}}^{*}+\mathscr{S}_{\widehat{\Sigma}}^{*}$.

\section{RIGHT-INVERSION}

All the results presented so far can be easily dualized, so as to turn a generic system $\Sigma$ into a right-invertible system $\widehat{\Sigma}$, without affecting its functional observability properties. Clearly, this transformation involves an output injection via a matrix $G$ such that (6) hold, and assigning all the eigenvalues

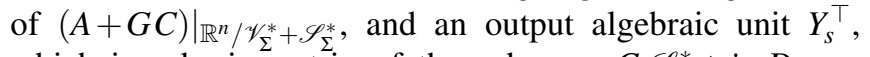
which is a basis matrix of the subspace $C \mathscr{S}_{\Sigma}^{*}+i m D$, see Fig. 2.

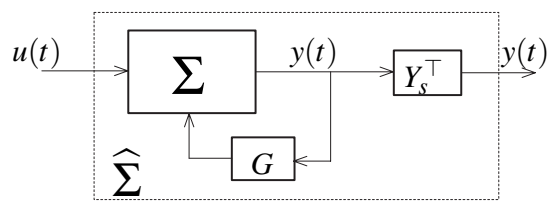

Fig. 2. Block diagram of a right-inverter

The following Corollary is the counterpart of Theorem 1 and Proposition 1 in the dual setting. 
Corollary 1: Let $G$ be a friend of $\mathscr{S}_{\Sigma}^{*}$. Let $Y_{S}$ be a basis matrix of the subspace $C \mathscr{S}_{\Sigma}^{*}+i m D$. The quadruple $\widehat{\Sigma}:=(A+$ $\left.G C, B+G D, Y_{s}^{\top} C, Y_{s}^{\top} D\right)$ is such that

1) $\mathscr{S}_{\Sigma}^{*}=\mathscr{S}_{\widehat{\Sigma}}^{*}$

2) $\widehat{\Sigma}$ is right-invertible;

3) $\mathscr{Z}(\widehat{\Sigma})=\left.\mathscr{Z}(\Sigma) \uplus \sigma(A+G C)\right|_{\mathbb{R}^{n} / \mathscr{V}_{\Sigma}^{*}+\mathscr{S}_{\Sigma}^{*}}$;

4) $\mathscr{V}_{\Sigma}^{i}+\mathscr{S}_{\Sigma}^{*}=\mathscr{V}_{\widehat{\Sigma}}^{i}+\mathscr{S}_{\widehat{\Sigma}}^{*}, i \in \mathbb{N}$. Hence, in particular, $\widehat{\Sigma}$ is left-invertible iff $\Sigma$ is such. Moreover, $\widehat{\Sigma}$ and $\Sigma$ have the same zero structure at infinity.

Hence, the set of invariant zeros of the resulting system $\widehat{\Sigma}$ is enlarged, so as to include the set of eigenvalues of $A+G C$ that are freely assignable through a suitable choice of $G$.

Furthermore, since the first transformation preserves the right-invertibility of the original system, while the second preserves the left-invertibility, these two procedures can be applied together to a generic system, possibly degenerate, so as to obtain a square and invertible system, described by the quadruple $\Sigma_{\mathrm{sq}}:=(A+B F+G C+G D F,(B+$ $\left.G D) U_{s}, Y_{s}^{\top}(C D F), Y_{s}^{\top} D U_{s}\right)$. Its set of invariant zeros includes the invariant zeros of the original system $\Sigma$. The matrices $F$ and $G$ can be chosen so as to place the additional invariant zeros introduced in the system $\Sigma_{\mathrm{sq}}$ thus obtained at arbitrary locations of the left half-plane $\mathbb{C}^{-}$, as pointed out in Theorem 1 and Proposition 1.

\section{A. A numerical example}

The proposed approach turns out to be easily implementable as a software routine for the squaring down of a generic LTI system. Consider the example described by the following matrices,

$$
\begin{aligned}
& A=\left[\begin{array}{cccc}
-5 & 0 & 20 & 0 \\
0 & -5 & 0 & 20 \\
0 & 0 & -8 & 0 \\
0 & 0 & 0 & -8
\end{array}\right], \quad B=\left[\begin{array}{ll}
0 & 0 \\
0 & 0 \\
1 & 0 \\
0 & 1
\end{array}\right], \\
& C=\left[\begin{array}{llll}
1 & 0 & 0 & 0 \\
0 & 0 & 1 & 0
\end{array}\right], \quad D=\left[\begin{array}{ll}
0 & 0 \\
0 & 0
\end{array}\right] .
\end{aligned}
$$

System $\Sigma=(A, B, C, D)$ is non left-invertible and non rightinvertible, since

$$
\mathscr{V}_{\Sigma}^{*} \cap \mathscr{S}_{\Sigma}^{*}=i m\left[\begin{array}{cc}
0 & 0 \\
1 & 0 \\
0 & 0 \\
0 & 1
\end{array}\right], \quad \mathscr{V}_{\Sigma}^{*}+\mathscr{S}_{\Sigma}^{*}=i m\left[\begin{array}{ccc}
0 & 0 & 0 \\
1 & 0 & 0 \\
0 & 0 & 1 \\
0 & 1 & 0
\end{array}\right] \text {. }
$$

In the present case $\mathscr{R}_{\Sigma}^{*}=\mathscr{V}_{\Sigma}^{*} \cap \mathscr{S}_{\Sigma}^{*}=\mathscr{V}_{\Sigma}^{*}$, so that $\Sigma$ has no invariant zeros. The first step consists of finding a friend $F \in \mathbb{R}^{2 \times 4}$ of $\mathscr{V}_{\Sigma}^{*}$ assigning the eigenvalues of $(A+B F)$ restricted to $\mathscr{R}_{\Sigma}^{*}$. The matrix

$$
F=\left[\begin{array}{cccc}
-0.1 & 0 & 6 & 0 \\
0 & -0.6 & 0 & 10
\end{array}\right]
$$

assigns the eigenvalues $\left.\sigma(A+B F)\right|_{\mathscr{R}_{\Sigma}^{*}}=\{-1,-2\}$, and $\left.\sigma(A+B F)\right|_{\mathbb{R}^{n} / \mathscr{V}_{\Sigma}^{*}+\mathscr{R}}=\{-3,-4\}$, where $\mathscr{R}$ is the reachable subspace from the origin, i.e., the smallest $A$ invariant subspace containing the range of $B$. The matrix
$U_{s}=\left[\begin{array}{ll}-1 & 0\end{array}\right]^{\top}$ is a basis matrix of $\left(B^{-1} \mathscr{V}_{\Sigma}^{*} \cap \operatorname{ker} D\right)^{\perp}$. The new system $\Sigma_{1}$ described by the matrices $A_{1}=A+B F$, $B_{1}=B U_{s}, C_{1}=C, D_{1}=D U_{s}=0$ is left-invertible, but not right-invertible, since $\mathscr{V}_{\Sigma_{1}}^{*}+\mathscr{S}_{\Sigma_{1}}^{*}=\mathscr{V}_{\Sigma}^{*}+\mathscr{S}_{\Sigma}^{*} \subset \mathbb{R}^{n}$. The set of its invariant zeros is exactly $\{-1,-2\}$. Now, we derive a friend $G \in \mathbb{R}^{4 \times 2}$ of $\mathscr{S}_{\Sigma}^{*}$ such that $\left.\sigma\left(A_{1}+G C_{1}\right)\right|_{\mathbb{R}^{n} / \mathscr{V}_{\Sigma}^{*}+\mathscr{S}_{\Sigma}^{*}}=$ $\{-4\}$ and $\left.\sigma\left(A_{1}+G C_{1}\right)\right|_{\mathscr{S}_{\Sigma}^{*} / \mathscr{S}_{\Sigma}^{*} \cap \mathscr{Q}}=\{-2\}$ :

$$
G=\left[\begin{array}{cc}
3 & -20 \\
0 & 0 \\
0 & -2 \\
0 & 0
\end{array}\right] .
$$

The matrix $Y_{s}=\left[\begin{array}{cc}0 & -1\end{array}\right]^{\top}$ is a basis matrix of $C_{1} \mathscr{S}_{\Sigma_{1}}^{*}+i m D$. The system $\Sigma_{2}$ described by the matrices $A_{2}:=A_{1}+G C_{1}, B_{2}:=B_{1}, C_{2}:=Y_{s}^{\top} C_{1}, D_{2}:=Y_{s}^{\top} D_{1}:$

$$
\begin{aligned}
& A_{2}=\left[\begin{array}{cccc}
-2 & 0 & 0 & 0 \\
0 & -5 & 0 & 20 \\
-0.1 & 0 & -4 & 0 \\
0 & -0.6 & 0 & 2
\end{array}\right], \quad B_{2}=\left[\begin{array}{c}
0 \\
0 \\
-1 \\
0
\end{array}\right], \\
& C_{2}=\left[\begin{array}{llll}
0 & 0 & -1 & 0
\end{array}\right], \quad D_{2}=0,
\end{aligned}
$$

is therefore left and right-invertible, since $\mathscr{R}_{\Sigma_{2}}^{*}=0_{n}$ and $\mathscr{V}_{\Sigma_{2}}^{*}+\mathscr{S}_{\Sigma_{2}}^{*}=\mathbb{R}^{n}$, and is minimum-phase, since its invariant zeros are $\{-1,-2,-2\}$.

\section{THE INFINITE-HORIZON LQ REGULATOR AND THE GENERALIZED DISCRETE ARE}

An interesting application of the results presented so far is the possibility of parametrizing the solutions of the infinitehorizon LQ problem for non left-invertible systems. Consider the discrete-time non left-invertible system $\Sigma$ described by

$$
\begin{aligned}
& x(k+1)=A x(k)+B u(k), \quad x(0)=x_{0} \in \mathbb{R}^{n}, \\
& y(k)=C x(k)+D u(k) .
\end{aligned}
$$

The problem is that of finding a stabilizing state feedback $u(k)=-K x(k)$ minimizing the performance index

$$
J\left(x_{0}, u\right)=\sum_{k=0}^{\infty} y^{\top}(k) y(k) .
$$

In this case, the optimal solution is not unique. In fact, since in this case $\mathscr{R}_{\Sigma}^{*}$ differs from zero, there exist control functions yielding state trajectories corresponding to identically zero output functions. In other words, given an optimal control law $u^{o}(k)$, the set of optimal control functions is parametrized modulo the controls driving the state of $\Sigma$ on $\mathscr{R}_{\Sigma}^{*}$. Let $Q:=$ $C^{\top} C, S:=C^{\top} D$ and $R:=D^{\top} D$. Since $\Sigma$ is not left-invertible, the discrete ARE

$$
\begin{aligned}
P=A^{\top} P A-\left(A^{\top} P B+S\right) & \left(R+B^{\top} P B\right)^{-1} \\
& \cdot\left(B^{\top} P A+S^{\top}\right)+Q,
\end{aligned}
$$

does not admit a stabilizing solution. Now, consider the system $\widehat{\Sigma}=(\widehat{A}, \widehat{B}, \widehat{C}, \widehat{D}):=\left(A+B F, B U_{s}, C+D F, D U_{s}\right)$, where $F$ is a friend of $\mathscr{V}_{\Sigma}^{*}$ and $U_{s}$ is a basis matrix of the subspace $\left(B^{-1} \mathscr{V}_{\Sigma}^{*} \cap k e r D\right)^{\perp}$. System $\widehat{\Sigma}$ is now left-invertible by virtue of Theorem 1. Hence, the infinite-horizon LQ problem can be solved with respect to the auxiliary system $\widehat{\Sigma}$ : in particular, 
the stabilizing solution $\widehat{P}$ of the discrete ARE (13) referred to $\widehat{\Sigma}$ yields the optimal gain $\widehat{K}:=\left(\widehat{R}+\widehat{B}^{\top} \widehat{P} \widehat{B}\right)^{-1}\left(\widehat{B}^{\top} \widehat{P} \widehat{A}+\widehat{S}^{\top}\right)$, where $\widehat{Q}:=\widehat{C}^{\top} \widehat{C}, \widehat{S}:=\widehat{C}^{\top} \widehat{D}$ and $\widehat{R}:=\widehat{D}^{\top} \widehat{D}$. Then, the matrix $K=U_{s} \hat{K}-F$ is a gain leading to an optimal control function. Different choices of the matrix $F$ yield different solutions of the LQ problem, corresponding to the possibility of assigning arbitrarily the eigenvalues of $(A+B F)$ on $\mathscr{R}_{\Sigma}^{*}$.

Thus, $\widehat{P}$ is not a solution of the discrete ARE since $R+B^{\top} \widehat{P} B$ is singular, but it a stabilizing solution of the so-called generalized discrete ARE

$$
\begin{aligned}
P=A^{\top} P A-\left(A^{\top} P B+S\right) & \left(R+B^{\top} P B\right)^{\ddagger} \\
& \cdot\left(B^{\top} P A+S^{\top}\right)+Q,
\end{aligned}
$$

Hence, the proposed method yields a non-iterative algorithm for the computation of the stabilizing solution of the generalized discrete ARE, as the following example will illustrate.

\section{A. An illustrative example}

Consider a discrete-time system $\Sigma$ described by

$$
\begin{array}{ll}
A=\left[\begin{array}{ll}
1 & 1 \\
0 & 1
\end{array}\right], \quad B=\left[\begin{array}{ll}
2 & 0 \\
1 & 1
\end{array}\right], \quad x(0)=\left[\begin{array}{c}
1 \\
-1
\end{array}\right], \\
C=\left[\begin{array}{ll}
0 & 1
\end{array}\right], \quad D=\left[\begin{array}{ll}
0 & 0
\end{array}\right],
\end{array}
$$

This system is non left-invertible, since $\mathscr{R}_{\Sigma}^{*}=i m\left[\begin{array}{ll}1 & 0\end{array}\right]^{\top}$, but it is right-invertible since $\mathscr{S}_{\Sigma}^{*}=\mathbb{R}^{n}$. Let $Q:=C^{\dagger} C$, $S:=C^{\top} D$ and $R:=D^{\top} D$. Since $\Sigma$ is not left-invertible, the discrete ARE does not admit a stabilizing solution. Consider the matrices

$$
F=\left[\begin{array}{cc}
-\frac{3}{4} & -\frac{1}{4} \\
\frac{3}{4} & -\frac{1}{4}
\end{array}\right] \quad U_{s}=\left[\begin{array}{l}
1 \\
1
\end{array}\right]
$$

where $F$ is a friend of $\mathscr{V}_{\Sigma}^{*}$, assigning $\left.\sigma(A+B F)\right|_{\mathscr{R}_{\Sigma}^{*}}=\left\{-\frac{1}{2}\right\}$ and $\left.\sigma(A+B F)\right|_{\mathbb{R}^{n} / \mathscr{N}_{\Sigma}^{*}+\mathscr{R}}=\left\{\frac{1}{2}\right\}$, while $U_{s}$ is a basis matrix for $\left(B^{-1} \mathscr{V}_{\Sigma}^{*}\right)^{\perp}$. The new system described by

$$
\begin{aligned}
& \widehat{A}=A+B F=\left[\begin{array}{cc}
-\frac{1}{2} & \frac{1}{2} \\
0 & \frac{1}{2}
\end{array}\right], \quad \widehat{B}=B U_{s}=\left[\begin{array}{l}
2 \\
2
\end{array}\right], \\
& \widehat{C}=C+D F=\left[\begin{array}{ll}
0 & 1
\end{array}\right], \quad \widehat{D}=D U_{s}=\left[\begin{array}{l}
0
\end{array}\right]
\end{aligned}
$$

is now left-invertible. The discrete ARE written with respect to $\widehat{A}, \widehat{B}, \widehat{Q}:=\widehat{C}^{\top} \widehat{C}, \widehat{S}:=\widehat{C}^{\top} \widehat{D}$ and $\widehat{R}:=\widehat{D}^{\top} \widehat{D}$, admits a stabilizing solution and an optimal infinite-horizon gain, which are respectively

$$
\widehat{P}=\left[\begin{array}{ll}
0 & 0 \\
0 & 1
\end{array}\right], \quad \widehat{K}=\left[\begin{array}{ll}
0 & \frac{1}{4}
\end{array}\right] .
$$

The optimal gain referred to the original system is

$$
K=U_{s} \widehat{K}-F=\left[\begin{array}{cc}
\frac{3}{4} & \frac{1}{2} \\
-\frac{3}{4} & \frac{1}{2}
\end{array}\right] .
$$

The overall optimal cost is $J^{o}=x_{0}^{\top} \widehat{P} x_{0}=1$. As we could expect, if we change $F$ in order to choose another eigenvalue of $A+B F$ restricted to $\mathscr{R}_{\Sigma}^{*}$ (for example $-\frac{1}{5}$ as internal and $-\frac{1}{2}$ as external assignable eigenvalues of $\mathscr{V}_{\Sigma}^{*}$, respectively), we find the same solution $\widehat{P}$ of (13) applied to $\widehat{\Sigma}$, leading to the same cost, with a different solution $\widehat{K}$. Hence, the optimal gain referred to the original system is, in this case,

$$
K^{\prime}=U_{s} \widehat{K}-F=\left[\begin{array}{cc}
\frac{3}{5} & 1 \\
-\frac{3}{5} & 1
\end{array}\right] \text {. }
$$

Finally, notice that $\widehat{P}$ is a stabilizing solution of the generalized discrete ARE.

\section{CONCLUDING REMARKS}

A geometric setting has been established for the solution of the left and right-inversion of non strictly proper LTI systems, leading to a simple and computationally attractive procedure for the squaring down problem. It has been proved that the squared-down system is minimum-phase if so is the original system, and the invariant zeros induced by the pre and postcompensators are all freely assignable in the complex plane. These new techniques are not merely oriented to the solution of the aforementioned problems, but highlight important geometric properties of LTI systems that can be exploited for the solution of different control problems; as an example we have considered the infinite-horizon LQ problem for discretetime non left-invertible systems, and the problem of finding a non iterative algorithm for the solution of the generalized discrete ARE.

\section{ACKNOWLEDGMENTS}

This research has been partially supported by the ministry of higher education of Italy (MIUR), under project Identification and Control of Industrial Systems.

\section{REFERENCES}

[1] H. Aling and J.M. Schumacher. A nine-fold canonical decomposition for linear systems. Int. J. of Control, 39(4):779-805, 1984.

[2] J.D. Aplevich. On the squaring problem in feedback design. In Proc. of the Joint Automatic Control Conference, vol. 43.6, pages WP-4P, Charlottesville 1981.

[3] G. Basile and G. Marro. A new characterization of some structural properties of linear systems: unknown-input observability, invertibility and functional controllability. Int. J. Control, 17(5):931-943, May 1973.

[4] G. Basile and G. Marro. Controlled and Conditioned Invariants in Linear System Theory. Prentice Hall, Englewood Cliffs, New Jersey, 1992.

[5] D. Chu and Y.S. Hung. A numerically reliable solution for the squaring-down problem in system design. Applied Numerical Mathematics, 51:221-241, 2004.

[6] E.J. Davison. Some properties of minimum-phase systems and 'squared-down' systems. IEEE Trans. Autom. Control, AC-28, 1983.

[7] V.X. Le and M.G. Safonov. Rational matrix GCD's and the design of squaring-down compensators - a state space theory. IEEE Trans. Autom. Control, AC-37(3):384-392, 1992.

[8] A.G.J. MacFarlane and N.Karcanias. Poles and zeros of linear multivariable systems: a survey of the algebraic, geometric and complexvariable theory. Int. J. Control, 24:33-74, 1976.

[9] A. Saberi and P. Sannuti. Squaring down of non-strictly proper systems. Int. J. Control, 51(3):621-629, 1990.

[10] P. Sannuti and A. Saberi. Squaring down by static and dynamic compensators. IEEE Trans. Autom. Control, AC-33(4):358-365, 1988.

[11] A.A. Stoorvogel and J.H.A. Ludlage. Squaring down and the problems of almost zeros for continuous-time systems. Systems \& Control Letters, 23(5):381-388, 1994.

[12] H.L. Trentelman, A.A. Stoorvogel, and M. Hautus. Control theory for linear systems. Communications and Control Engineering. Springer, Great Britain, 2001.

[13] Wonham, W.M. Linear Multivariable Control: A Geometric Approach. Springer-Verlag, New York, 1985. 